\title{
Photocrosslinkable Gelatin/Tropoelastin Hydrogel Adhesives for Peripheral Nerve Repair
}

\author{
Jonathan R. Soucy, BS, Ehsan Shirzaei Sani, MS,, Roberto Portillo Lara, PhD,,2 David Diaz, Felipe Dias,' \\ Anthony S. Weiss, PhD, Abigail N. Koppes, PhD, ${ }^{1,4}$ Ryan A. Koppes, PhD,' and Nasim Annabi, PhD 1,5,6
}

Suturing peripheral nerve transections is the predominant therapeutic strategy for nerve repair. However, the use of sutures leads to scar tissue formation, hinders nerve regeneration, and prevents functional recovery. Fibrinbased adhesives have been widely used for nerve reconstruction, but their limited adhesive and mechanical strength and inability to promote nerve regeneration hamper their utility as a stand-alone intervention. To overcome these challenges, we engineered composite hydrogels that are neurosupportive and possess strong tissue adhesion. These composites were synthesized by photocrosslinking two naturally derived polymers, gelatin-methacryloyl (GelMA) and methacryloyl-substituted tropoelastin (MeTro). The engineered materials exhibited tunable mechanical properties by varying the GelMA/MeTro ratio. In addition, GelMA/MeTro hydrogels exhibited 15-fold higher adhesive strength to nerve tissue ex vivo compared to fibrin control. Furthermore, the composites were shown to support Schwann cell (SC) viability and proliferation, as well as neurite extension and glial cell participation in vitro, which are essential cellular components for nerve regeneration. Finally, subcutaneously implanted GelMA/MeTro hydrogels exhibited slower degradation in vivo compared with pure GelMA, indicating its potential to support the growth of slowly regenerating nerves. Thus, GelMA/MeTro composites may be used as clinically relevant biomaterials to regenerate nerves and reduce the need for microsurgical suturing during nerve reconstruction.

Keywords: nerve anastomosis, nerve repair, Schwann cells, GelMA, MeTro

\section{Introduction}

$\mathbf{P}$ ERIPHERAL NERVE INJURY (PNI) often leads to partial or complete loss of sensation, chronic pain, apraxia, and even permanent disability. ${ }^{1}$ Due to the slow rate and limited ability of the peripheral nervous system (PNS) to regenerate, ${ }^{2}$ surgical intervention is often required for function recovery. Regardless of the type of surgical intervention for PNI [e.g., anastomosis (Fig. 1A) or grafting (Fig. 1B)], standard therapies most often involve the use of nylon or polypropylene sutures to mechanically stabilize and reconstruct nerves. ${ }^{3,4}$ However, the use of nylon or polypropylene sutures for nerve anastomosis may further damage the injured tissue resulting in increased tension, inflammation, and the development of a foreign body response (FBR). ${ }^{3-5}$ In particular, excess tension in neural tissues has been associated with reduced angiogenesis, which is essential for the promotion of tissue remodeling and axonal regeneration. ${ }^{6}$
Several sutureless interventions have been proposed, including tissue laser welding (LTW), ${ }^{7,8}$ photochemical bonding (PTB), ${ }^{9,10}$ as well as the use of bioadhesives. ${ }^{11-14}$ While promising, these strategies have a number of limitations for PNS repair. LTW can thermally induce damage to proximal nerve fibers, slowing functional recovery. Furthermore, LTW often requires suturing to mechanically stabilize the nerve. In contrast, PTB can only be utilized for nongap and critical size injuries. ${ }^{12}$ Neural glues are an attractive approach to overcome these limitations, but current compositions and formulations have not met the mechanical and adhesive properties required for sutureless repair. Specifically, fibrinbased glues have low stiffness and adhesion and high risk of infection, ${ }^{15,16}$ cyanoacrylate glues lack biocompatibility and often elicit a FBR, ${ }^{17,18}$ and polyethylene glycol-based tissue bonding degrades too slowly, persisting for years following implantation. ${ }^{12,13}$ Therefore, an elastic and biocompatible biomaterial with appropriate tissue adhesion and

\footnotetext{
${ }^{1}$ Department of Chemical Engineering, Northeastern University, Boston, Massachusetts.

${ }^{2}$ Tecnológico de Monterrey, Escuela de IngenierÍa y Ciencias, Zapopan, JAL, Mexico.

${ }^{3}$ Charles Perkins Centre, School of Life and Environmental Sciences and Bosch Institute, University of Sydney, Sydney, Australia.

${ }^{4}$ Department of Biology, Northeastern University, Boston, Massachusetts.

${ }^{5}$ Harvard-MIT Division of Health Sciences and Technology, Massachusetts Institute of Technology, Cambridge, Massachusetts.

${ }^{6}$ Department of Chemical and Biomolecular Engineering, University of California, Los Angeles, Los Angeles, California.
} 

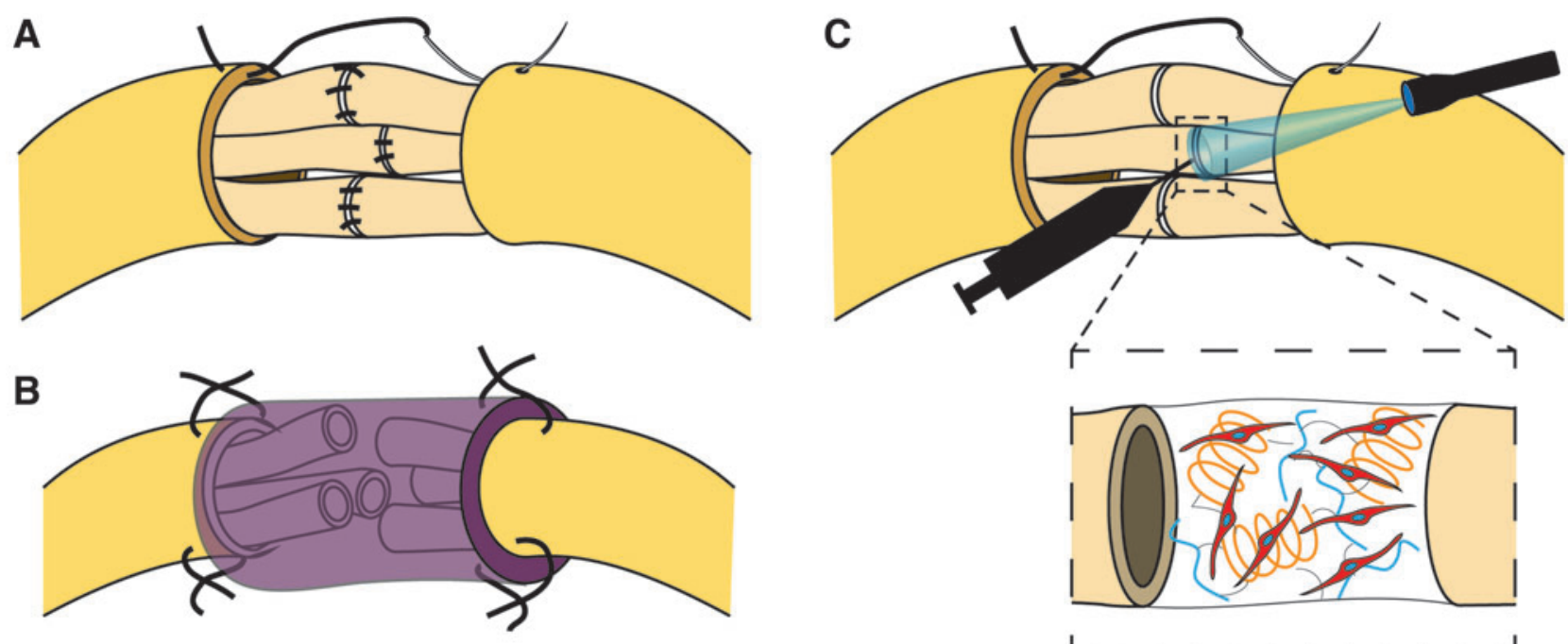

D

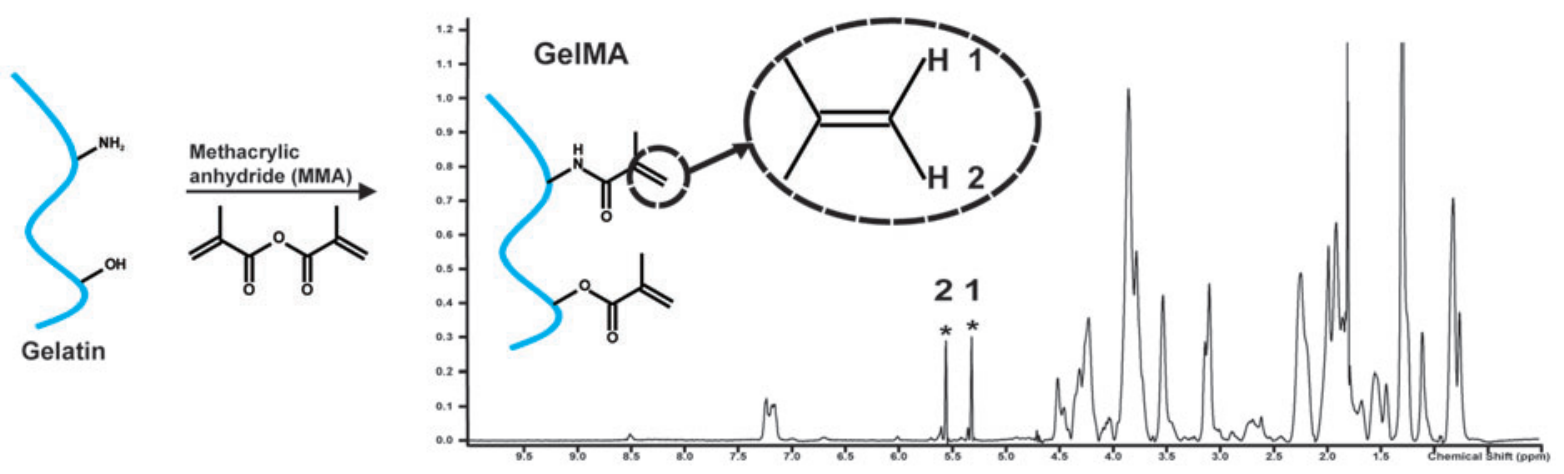

$\mathbf{E}$
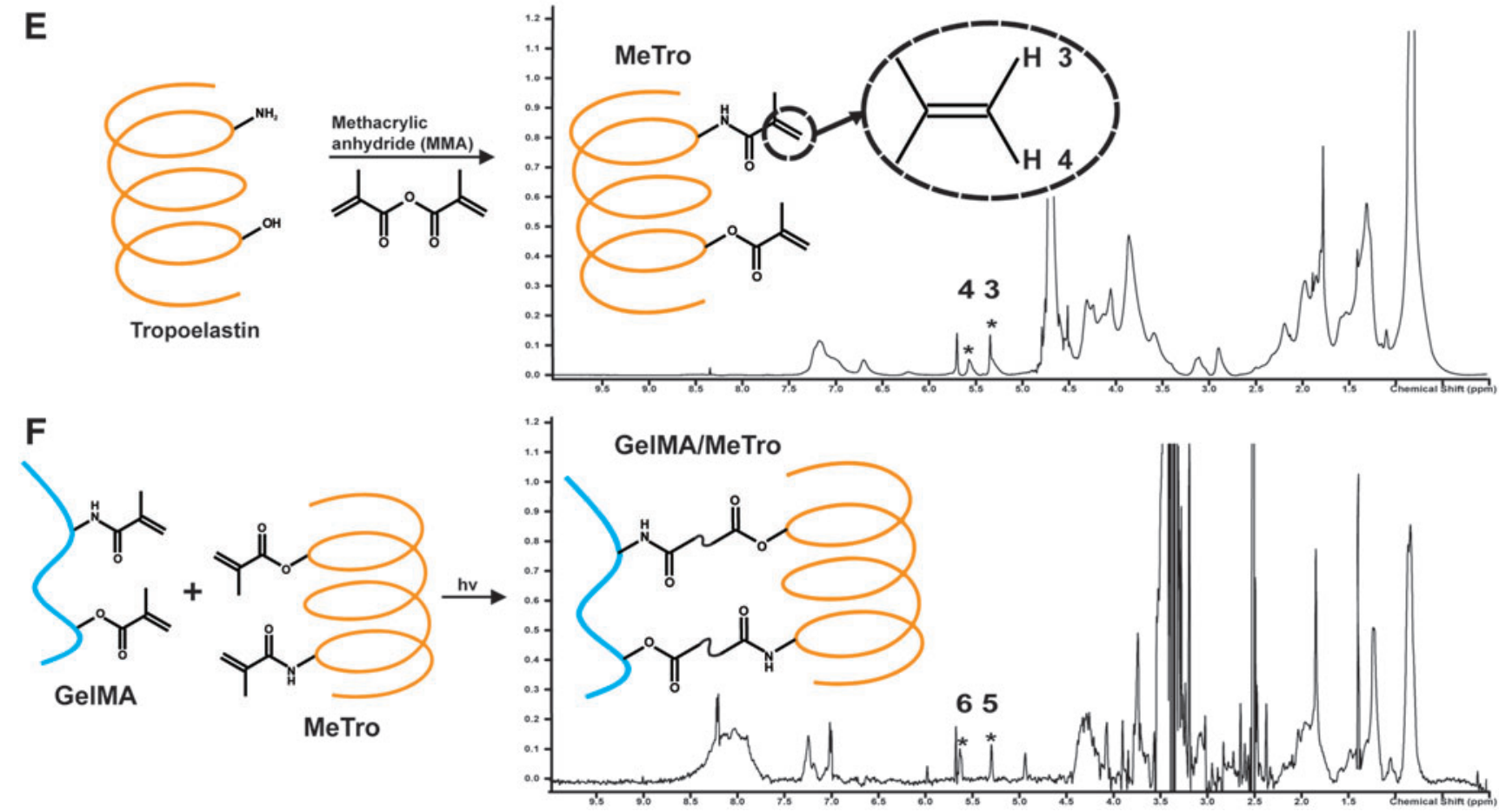

FIG. 1. GelMA/MeTro composite hydrogel application and chemical validation. (A) Transected nerve repaired by suturing individual fascicles and the connective tissue. (B) Transected nerve repaired with a sutured-on hollow nerve conduit. (C) Transected nerve repaired by photocrosslinking a SC-laden GelMA/MeTro composite adhesive to align fascicles and suturing of connective tissue. The use of engineered adhesive limits the total number of sutures. Proton nuclear magnetic resonance (D) GelMA, (E) MeTro, and (F) GelMA/MeTro composite confirming the degree of crosslinking in the composite to be 73.3\%. SC, Schwann cell; GelMA, gelatin-methacryloyl; MeTro, methacryloyl-substituted tropoelastin. Color images available online at www.liebertpub.com/tea 
stiffness that can also support neurite outgrowth may be advantageous to overcome the limitations of current sutureless repair strategies.

Herein, we developed a photocrosslinkable composite of gelatin-methacryloyl (GelMA) and methacryloyl-substituted tropoelastin (MeTro) hydrogels (Fig. 1C) with tunable physicochemical properties for neural tissue engineering applications. The incorporation of two biopolymers with distinctive biophysical and biochemical characteristics enabled the engineering of hydrogels with a wide spectrum of physicochemical properties. We evaluated the physical properties of the engineered hydrogels, including porosity, stiffness, elasticity, swellability, and in vitro and in vivo degradation rates. In addition, we examined the efficacy of the engineered GelMA/ MeTro composite to act as an adhesive for nerve anastomosis ex vivo. We also investigated the ability of this material to support the viability and proliferation of Schwann cells (SCs) and extension of PNS neurons encapsulated within the composite hydrogels. Finally, we evaluated the biodegradability and immunogenicity of the engineered hydrogels when implanted subcutaneously in vivo. We hypothesize that the engineered GelMA/MeTro hydrogel may be used as a multifunctional adhesive with regenerative capabilities for nerve repair and anastomosis.

\section{Materials and Methods}

\section{GelMA and MeTro synthesis}

GelMA was synthesized by adding $8 \%$ (v/v) methacrylic anhydride (MA; Sigma) to a $10 \%$ (w/v) fish gelatin solution in Dulbecco's phosphate buffered saline (DPBS; Sigma), as previously described. ${ }^{19}$ This reaction was carried out at $60^{\circ} \mathrm{C}$ for $2 \mathrm{~h}$ and stopped by adding a $3 \times$ dilution of warm $\left(60^{\circ} \mathrm{C}\right)$ DPBS. The mixture was dialyzed (Spectra/Por $12-14 \mathrm{kD}$; Fisher Scientific) using distilled water for 1 week at $60^{\circ} \mathrm{C}$, frozen at $-80^{\circ} \mathrm{C}$, lyophilized, and stored at room temperature for use on demand.

Similarly, a 10\% (w/v) tropoelastin isoform (SHEL $\Delta 26 \mathrm{~A}$, synthetic human elastin without amino acid residues $27-724$ of GenBank entry AAC98394) solution was mixed with $8 \%$ (v/v) MA to form MeTro, as previously reported. ${ }^{20}$ The reaction was carried out for $12 \mathrm{~h}$ at $4^{\circ} \mathrm{C}$ and then stopped by adding a $3 \times$ dilution of cold $\left(4^{\circ} \mathrm{C}\right)$ DPBS. The diluted solution was then dialyzed (Slide-A-Lyzer Dialysis Cassette; $3500 \mathrm{MWCO})$ for $48 \mathrm{~h}$ in distilled water at $4^{\circ} \mathrm{C}$. The resulting MeTro was frozen at $-80^{\circ} \mathrm{C}$, lyophilized, and stored at room temperature for on demand use.

\section{Hydrogel fabrication}

Composite hydrogels were synthesized of varying ratios of GelMA and MeTro and different total polymer concentrations. Hydrogel precursor solution was prepared in distilled water with $0.5 \%$ (w/v) 2-hydroxy-1-(4-(hydroxyethoxy) phenyl)-2methyl-1-propanone (Irgacure 2959; CIBA Chemicals). To ensure a homogenous precursor solution, the mixture was placed on ice for $15 \mathrm{~min}$ until both polymers dissolved completely. The solution was then photocrosslinked $\left(6.9 \mathrm{~mW} / \mathrm{cm}^{2}\right.$ of UV light [320-390 nm]) for $180 \mathrm{~s}$ for all material characterizations and $35 \mathrm{~s}$ for the in vitro cell studies $(0.23 \mathrm{~s}$ of exposure per $\mu \mathrm{m}$ of gel thickness).

\section{Proton nuclear magnetic resonance analysis}

Spectra were acquired with a Varian Inova-500 proton nuclear magnetic resonance $\left({ }^{1} \mathrm{H}\right.$ NMR) spectrometer for uncrosslinked GelMA and MeTro dissolved in deuterium oxide $\left(\mathrm{D}_{2} \mathrm{O}\right)$ and the supernatant from a partially dissolved composite (50/50 GelMA/MeTro, 10\% [w/v] total polymer concentration) kept in deuterated dimethyl sulfoxide (DMSO-d6; Sigma) overnight at room temperature. The degree of crosslinking was calculated by the following equation:

$$
\text { Degree of crosslinking }(\%)=\left(\mathrm{PA}_{\mathrm{b}}-\mathrm{PA}_{\mathrm{a}}\right) / \mathrm{PA}_{\mathrm{b}} \times 100,{ }^{21}
$$

where $\mathrm{PA}_{\mathrm{b}}$ is equal to the peak area before crosslinking, and $\mathrm{PA}_{\mathrm{a}}$ is the peak area after crosslinking. Peak areas were measured using ACD/Spectrus NMR to integrate the area of the curve with respect to phenolic conjugated peaks at $\delta=6.5-7.5 \mathrm{ppm}$.

\section{Mechanical characterization}

Compressive and tensile properties of hydrogel formulations were measured at a $1 \mathrm{~mm} / \mathrm{min}$ strain rate using a universal load frame (Instron Model 5542). Hydrogel samples were prepared in custom polydimethylsiloxane (PDMS; Sylgard) molds (cylinders of $6.30 \mathrm{~mm}$ diameter by $1.50 \mathrm{~mm}$ depth for compressive testing; and cuboids of $12.40 \mathrm{~mm}$ length, $5.00 \mathrm{~mm}$ width, and $0.80 \mathrm{~mm}$ depth for tensile testing). Samples were allowed to swell in DPBS for $2 \mathrm{~h}$ at $37^{\circ} \mathrm{C}$ before mechanical testing. Moduli were calculated as the tangent slope of the linear region (between 0.1 and 0.25 strain level) of the respective stress-strain curves. Energy loss was calculated from cyclic compression tests as the area between the loading and unloading curves for cycle 8 . The ultimate stress and extensibility were measured as the maximum stress and strain/extensibility at tensile failure, respectively. We investigated the effect of the GelMA/ MeTro ratio, final polymer concentration, and light exposure time on the mechanical properties of the hydrogels using at least three independent samples for each set of conditions. The results are shown as the average \pm standard deviation.

\section{Scanning electron microscopy analysis}

All samples were lyophilized, mounted on aluminum pin stubs, and sputter coated with a $6 \mathrm{~nm}$ gold/palladium ( $\mathrm{Au} /$ Pd) layer. Scanning electron microscopy (SEM) images were acquired using a Hitachi S-4800 SEM at $15 \mathrm{kV}$ and an $8 \mathrm{~mm}$ working distance. GelMA/MeTro hydrogels were all processed in the same conditions, based on a method reported previously in the literature. ${ }^{22}$

\section{Swelling ratio and in vitro degradation characterization}

Hydrogel samples were frozen at $-80^{\circ} \mathrm{C}$, lyophilized, and weighed to determine their dry weights. To measure swelling, hydrogels were soaked in DPBS at $37^{\circ} \mathrm{C}$ for different time points $(1,2,4,8,12,24 \mathrm{~h})$ and reweighed. Swelling ratios were calculated as the change between the wet and dry weights over the dry weight. For the in vitro degradation study, each sample was kept in $1 \mathrm{~mL}$ of $5 \%$ (v/v) fetal bovine serum (FBS; Corning) in DPBS at $37^{\circ} \mathrm{C}$ for 1,7 , or 14 days. At these established time points, the 
solutions were removed, and the samples were frozen, lyophilized, and weighed again. The percentage of degradation was calculated as a ratio of the final and initial dry weights of the hydrogels.

\section{Nerve anastomosis}

Sciatic nerve explants $(\sim 18 \mathrm{~mm})$, proximal to the bifurcation at the knee and distal from the spinal column, were dissected from the legs of adult rats (male Wistar [200-250 g] and female Sprague Dawley [250-350g]). To maintain cell viability and structural stability, intact nerve sections were stored in Hibernate ${ }^{\circledR}$-A on ice for $4-12 \mathrm{~h}$ before adhesion testing to allow time for material preparation. ${ }^{23,24}$ On a Teflon ${ }^{\circledR}$ surface, each nerve was attached with super glue between two pieces of 150 grit sandpaper with $\sim 12 \mathrm{~mm}$ gauge length. Using titanium clamps, nerves were then secured in a Bose ElectroForce 3200ES. Nerve trunks were transected with surgical scissors and reconnected through the application of $\sim 5 \mu \mathrm{L}$ of $50 / 50 \mathrm{Gel}-$ $\mathrm{MA} / \mathrm{MeTro}$ precursor solution at $10 \%(\mathrm{w} / \mathrm{v})$ total polymer concentration and direct exposure to UV light for $180 \mathrm{~s}$ or with $\sim 5 \mu \mathrm{L} \mathrm{EVICEL}^{\circledR}$, a commercially available fibrin-based adhesive. Both the nerve and hydrogel were hydrated with DPBS before being pulled at a rate of $0.08 \mathrm{~mm} / \mathrm{s}$ until failure to recapitulate an in vivo environment. The load and displacement data were recorded at a sampling rate of $20 \mathrm{~Hz}$. Each hydrated nerve was imaged and analyzed by ImageJ to measure its diameter to determine the cross-sectional area before testing. The adhesion strength was measured as the stress at failure. To observe the adhesive-tissue interface, the nerves, anastomosed by the engineered adhesives, were fixed in $4 \%$ paraformaldehyde (Sigma) in DPBS for $4 \mathrm{~h}$, kept overnight in DPBS containing $30 \%$ sucrose at $4^{\circ} \mathrm{C}$, embedded in Optimal Cutting Temperature (OCT) compound, and flash frozen. The fixed samples were then cryo-sectioned ( $15 \mu \mathrm{m}$ slices), and hematoxylin and eosin (H\&E; Sigma) staining was performed to observe adhesive/tissue interface.

\section{Three dimensional encapsulation of SCs inside the hydrogels}

Primary SCs were isolated from 2-day-old (p2) Sprague Dawley neonatal rat (Charles River) sciatic nerves, as previously reported. ${ }^{25-27} \mathrm{SCs}$ were purified with an antimitotic and a rabbit complement CD90 targeted cell lysis. SC purity was assessed using immunofluorescence and found to be $>95 \%$ (data not shown). SCs $(<\mathrm{P} 10)$ were mixed with the hydrogel precursor solution containing 80/20 GelMA/MeTro (10\% [w/ v] total polymer concentration) at a density of $5 \times 10^{6}$ cells $/ \mathrm{mL}$. Approximately $7 \mu \mathrm{L}$ cell-laden gel precursor solution was placed between a $150 \mu \mathrm{m}$-tall spacer and a 3-(trimethoxysilyl) propyl methacrylate (ACROS Organics) coated glass slide followed by exposure to light to form cell-laden GelMA/MeTro hydrogels. Samples were incubated in standard culture conditions $\left(37^{\circ} \mathrm{C}, 5 \% \mathrm{CO}_{2}\right)$ for 1,3 , and 5 days, and $\mathrm{SC}$ maintenance medium (Dulbecco's Modified Eagle's Medium [Corning] supplemented with 10\% FBS, $2 \mathrm{mM}$ L-glutamine [Gibco], 50 U/mL penicillin/streptomycin [P/S; Gibco], $6.6 \mathrm{mM}$ forskolin [Sigma], and $10 \mu \mathrm{g} / \mathrm{mL}$ bovine pituitary extract [Corning]) was changed every other day. SC viability was determined using a LIVE/DEAD ${ }^{\circledR}$ Viability/Cytotoxicity Kit (Life Technologies) and quantified as the number of the live cells (shown in green) divided by total cell (sum of green and red cells) number in a $10 \times$ magnification image using ImageJ software. The metabolic activity of SCs was evaluated using the PrestoBlue assay (Life Technologies) and a Synergy HT fluorescence plate reader (BioTek).

\section{Three dimensional encapsulation of whole dorsal root ganglia inside the hydrogels}

Dorsal root ganglia (DRGs), from the thoracic and lumbar regions of the spinal column, were isolated from p2 Sprague Dawley neonatal rats as per established protocols. ${ }^{23,28,29}$ DRGs were collected and randomized across three animals from two separate litters (total=six p2 pups) and were encapsulated in hydrogels in a similar manner to SCs (see section "Three dimensional encapsulation of SCs"). A single whole DRG was suspended in $\sim 10 \mu \mathrm{L}$ of a $50 / 50$ GelMA/MeTro (10\% [w/v] final polymer concentration) precursor solution and placed between a $300 \mu \mathrm{m}$-tall spacer. Samples were photocrosslinked and incubated in standard culture conditions for 7 days. DRG maintenance medium (Neurobasal medium [Gibco] supplemented with $1 \times$ B27 [Gibco], $2 \mathrm{mM} \mathrm{L}$-glut, $50 \mathrm{U} / \mathrm{mL} \mathrm{P} / \mathrm{S}$, and $25 \mathrm{ng} / \mathrm{mL}$ nerve growth factor [Gibco]) was changed every other day. DRG outgrowth was quantified by the total outgrowth area in MATLAB as previously reported. ${ }^{30}$

\section{Immunocytochemistry analysis (actin/4',6-diamidino-2- phenylindole/S100/B3T)}

Cell-laden hydrogels were fixed in $4 \%$ paraformaldehyde for $30 \mathrm{~min}$, permeabilized with $0.1 \%$ Triton X-100 (Sigma) for $30 \mathrm{~min}$ at room temperature, and blocked overnight at $4^{\circ} \mathrm{C}$ with 5\% goat serum (Sigma). After blocking, rabbit anti S100 (DAKO; Z0311) and/or mouse anti $\beta$-3 tubulin (Invitrogen; 480011) (1:400 in 5\% goat serum) were added and the samples were overnight at $4^{\circ} \mathrm{C}$. After primary antibody incubation, cell-laden hydrogels were washed with DPBS, and anti-rabbit and/or anti-mouse secondary antibodies (1:200 and phalloidin [Life Technologies; A22287] 1:400 in goat serum) were added and the hydrogels were kept at $4^{\circ} \mathrm{C}$ overnight. Samples were washed with DPBS, mounted on cover slides with ProLong ${ }^{\circledR}$ Gold Antifade with $4^{\prime}, 6$ diamidino-2-phenylindole (DAPI), and imaged using an inverted fluorescence microscope (Zeiss Axio Observer Z1).

\section{In vivo degradation and biocompatibility}

Male Wistar rats (200-250 g; Charles River) were anesthetized using 1-2.5\% isoflurane, and lyophilized hydrogel samples (50/50 GelMA/MeTro at 10\% [w/v] total polymer concentration), prepared in PDMS molds (cylinders $4.92 \mathrm{~mm}$ diameter, $3.85 \mathrm{~mm}$ height), were implanted subcutaneously, as previously described. ${ }^{31-33}$ On days 4,14 , 28 , and 56 post-implantation, rats were euthanized, and the hydrogels, along with the adjacent tissue, were explanted. Surrounding tissue was carefully removed for samples used to evaluate the degradation rate in vivo. These explants were then lyophilized overnight so that the final dry weight could be measured. Degradation ratios were reported as final dry weight/initial dry weight for each post-implantation time point. Following explantation, samples were fixed for $4 \mathrm{~h}$ in $4 \%$ paraformaldehyde in DPBS, incubated overnight at $4^{\circ} \mathrm{C}$ in 30\% sucrose in DPBS, embedded in OCT compound, and 
flash frozen in dry ice. Fifteen-micrometer cryosections were obtained using a Leica Biosystems CM3050S Research Cryostat. Immunohistological staining was performed as previously reported. ${ }^{34}$ Samples were stained for lymphocytes (1:100 Anti-CD3 [SP7], ab16669; Abcam) and macrophages $(1 \mu \mathrm{g} / \mathrm{mL}$ anti-CD68, ab125212; Abcam), detected using an Alexa Fluor 546-conjugated secondary antibody (Invitrogen; A11034) and counterstained with DAPI (Invitrogen). Cryosections were attached to positively charged slides and mounted using DPX mounting medium (Sigma), and images were acquired using an Axio Observer Z1 inverted microscope. Slides were processed for H\&E staining as per instructions from the manufacturer.

\section{Statistical analysis}

Data were compared using one-way analysis of variance test in GraphPad Prism software. Error bars represent the mean \pm standard deviation of measurements $(* p<0.05, * * p<$ $0.01, * * * p<0.001$, and $* * * * p<0.0001)$. For mechanical characterization, swelling ratio, in vitro and in vivo degradation, SC metabolic activity, SC viability (four images/ gel), DRG outgrowth, and histological staining (seven slides/ gel), a minimum of three independently prepared samples were used to determine the average and standard deviation. A total of six nerves were used for each nerve anastomosis condition.

\section{Animal use statement}

All animals were treated following Northeastern University's Institutional Animal Care and Use Committee approved protocols.

\section{Results}

\section{Synthesis and fabrication of GeIMA/MeTro hydrogels}

To engineer a multifunctional material for the treatment of PNI, recombinant human tropoelastin and gelatin were methacrylated to form the photocrosslinkable biopolymers GelMA $^{19}$ and MeTro. ${ }^{20}{ }^{1} \mathrm{H}$ NMR spectra were taken from dissolved GelMA (Fig. 1D) and MeTro (Fig. 1E) in $\mathrm{D}_{2} \mathrm{O}$ and partially dissolved GelMA/MeTro composite in DMSO-d6 (Fig. 1F) to quantify the degree of crosslinking. ${ }^{35}$ These results indicated that the methacrylate groups present in GelMA and MeTro were involved in the formation of the composite hydrogel. In these spectra, the methacrylate groups $\left(-\mathrm{C}=\mathrm{CH}_{2}\right)$ appeared as characteristic peaks at $\delta=5.3 \mathrm{ppm}$ (peak 1, 3, 5) and $\delta=5.7 \mathrm{ppm}$ (peak 2, 4, 6). The disappearance of peaks 5 and 6 , shown in the GelMA/MeTro spectra and normalized to phenolic conjugated peaks $(\delta=$ $6.5-7.5 \mathrm{ppm}$ ), indicated that the degree of crosslinking was $\sim 73.3 \%$ following exposure to light.

\section{Mechanical characterization of GelMA/MeTro hydrogel}

The mechanical properties of GelMA/MeTro hydrogels were tuned by varying the ratio of GelMA to MeTro (Fig. 2A-F), the total polymer concentration (Fig. S1A, B), and the crosslinking time (Supplementary Fig. S1C, D; Supplementary Data are available online at www.liebertpub .com/tea). Tensile tests showed statistically significant differences in the mechanical properties between the composites and pure GelMA and MeTro hydrogels (Fig. 2A-C).
Specifically, the tensile modulus, strain at failure, and ultimate stress of the composites decrease by increasing the GelMA/MeTro ratio (Fig. 2A-C). Based on the cyclic compression test, it was found that composites had compressive moduli greater than those of pure GelMA or MeTro hydrogels (Fig. 2D, E). Results also demonstrated that the compressive moduli of 50/50 GelMA/MeTro hydrogels increased concomitantly by increasing the total polymer concentration (Supplementary Fig. S1A, B). An increase in total polymer concentration of GelMA or MeTro hydrogels may result in a higher degree of crosslinking and, thus, increased modulus. ${ }^{19,20}$ In addition, the compressive moduli of the composites increased with increasing light exposure time (Supplementary Fig. S1C, D).

\section{Structural characteristics, swelling ratios, and in vitro degradation of GelMA/MeTro hydrogels}

The microstructure of hydrogels has been shown to influence cell infiltration and tissue integration. ${ }^{36}$ Analysis of hydrogel structure through SEM demonstrated the influence of varying ratios of GelMA and MeTro on the composite microarchitecture (Supplementary Fig. S2). While observable differences in hydrogel porosity and structure were apparent among different formulations, lyophilization of the samples before SEM analysis can also affect the porous structures of the freeze dried samples. ${ }^{36}$

Swellability is an important factor for material design, as increased permeability can improve the diffusion of nutrients, oxygen, and metabolic waste. ${ }^{37}$ Conversely, materials that exhibit limited swelling may be favorable for implantation, as materials with high levels of swelling may increase interstitial pressure at the injury site. ${ }^{38,39}$ Therefore, the swellability of GelMA/MeTro hydrogels was evaluated in a model physiological fluid. The swelling ratio of pure GelMA hydrogels was nearly five times that of MeTro after $24 \mathrm{~h}$ of incubation in DPBS, while the composites exhibited swelling ratios between that of each pure species (Fig. 2G). In addition, the swelling ratios for all tested GelMA/MeTro composites decreased by increasing the total polymer concentrations (Supplementary Fig. S3A), which were consistent with previously reported trends for pure GelMA hydrogels. ${ }^{40}$ Similarly, increasing the time of crosslinking from 30 to $60 \mathrm{~s}$ also resulted in a decrease in swelling ratio, which was approximately halved for all measured time points, likely due to increased crosslinking (Supplementary Fig. S3B). ${ }^{40,41}$

Swellability may also influence the rate of degradation for hydrogels in vitro. ${ }^{42}$ Increasing the concentration of GelMA in the composites led to an increase in the degradation rate in DPBS supplemented with 5\% FBS over 14 days (Fig. 2H). The degradation ratio of the composites increased from $3.85 \pm 3.55 \%$ for pure MeTro to $18.73 \pm 3.88 \%$ for pure GelMA after $24 \mathrm{~h}$ (Fig. $2 \mathrm{H}$ ). There was no significant difference between the degradation of the 50/50 GelMA/ MeTro $(5.48 \pm 4.78 \%)$ composite and that of pure MeTro.

\section{Adhesive strength of GelMA/MeTro composites for nerve anastomosis}

To recapitulate the ideal surgical intervention strategy currently utilized with fibrin and sutures, ${ }^{43}$ the severed proximal and distal nerve stumps of adult rat sciatic nerves 

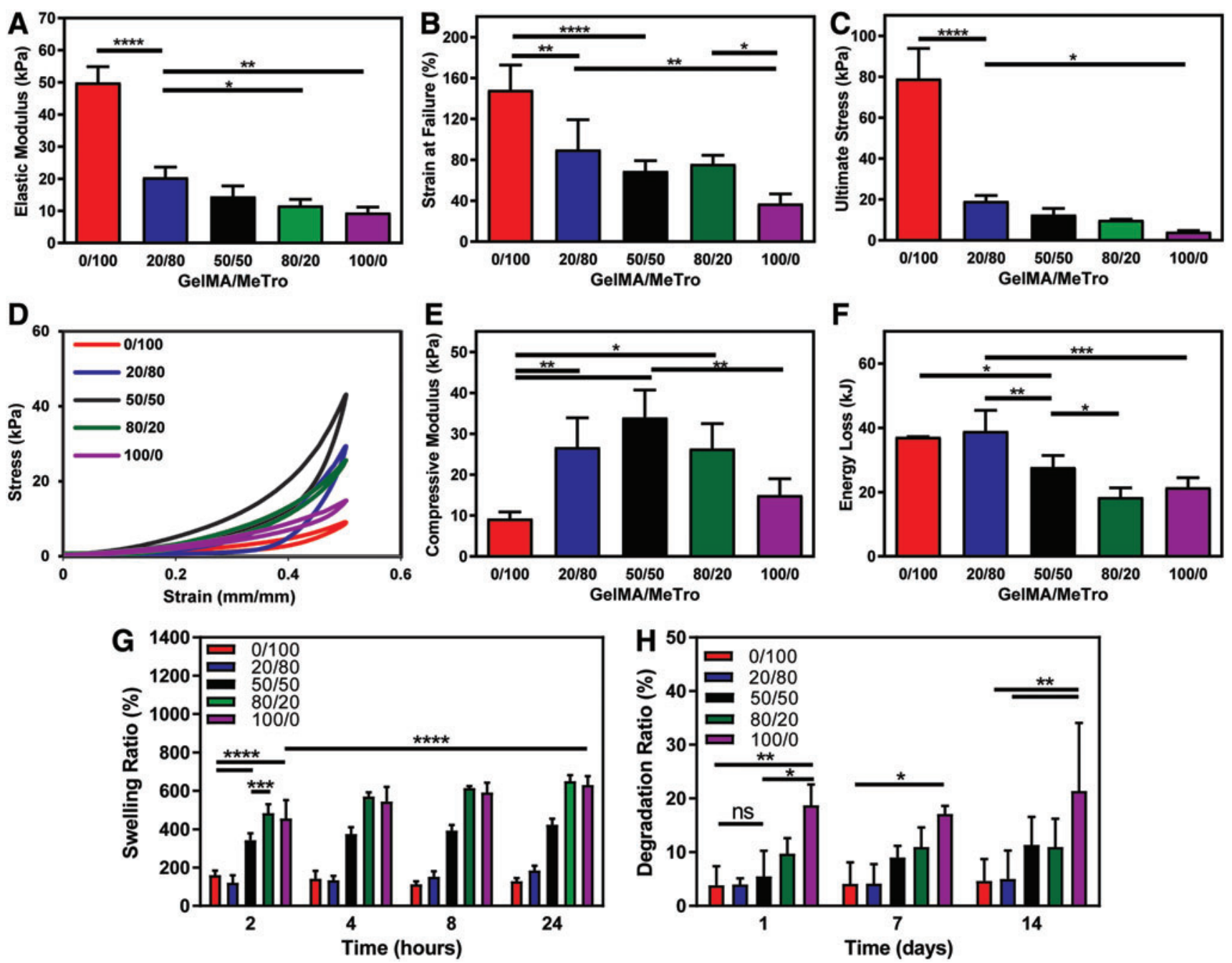

FIG. 2. Physical characterizations of GelMA/MeTro composite hydrogels. (A) Elastic modulus, (B) strain at failure, (C) ultimate stress of GelMA/MeTro hydrogels produced using various GelMA/MeTro ratios at 10\% (w/v) polymer concentration. (D) Representative compressive stress-strain curves, (E) compressive modulus, (F) energy loss of GelMA/MeTro hydrogels at varying GelMA/MeTro ratios and 10\% (w/v) total polymer concentrations. (G) Swelling ratio of the engineered hydrogels after 2, 4, 8, and $24 \mathrm{~h}$ incubation in DPBS, and (H) degradation ratios of hydrogels in 5\% (v/v) fetal bovine serum in DPBS on days 1, 7, and 14. All the hydrogels were formed using $180 \mathrm{~s}$ crosslinking time. (ns: $p>0.05$, $* p<0.05, * * p<0.01, * * * p<0.001, * * * * p<0.0001)$. DPBS, Dulbecco's phosphate buffered saline. Color images available online at www.liebertpub.com/tea

were connected end-to-end ex vivo using GelMA/MeTro and a commercially available fibrin-based glue, EVICEL. The adhesion tests (Fig. 3A) demonstrated a rapid and improved mechanical stabilization of severed nerve tissue glued by GelMA/MeTro over EVICEL ex vivo (Fig. 3B). Our results indicated that GelMA/MeTro composites exhibited a significantly higher adhesive strength $(165.2 \pm 25.8 \mathrm{kPa})$ than EVICEL $(10.3 \pm 3.4 \mathrm{kPa})$, when used for nerve anastomosis (Fig. 3B). In addition, the adhesive strength of GelMA/MeTro composites was two-fold higher than a previously proposed photocrosslinkable chitosan hydrogel for nerve repair $(71.3 \pm 23.4 \mathrm{kPa}){ }^{44}$ The strain at failure for ex vivo anastomosis was $14 \pm 3 \%$ (Fig. 3C), which was higher than the value reported for the permanent nerve dysfunction $(\sim 10 \%$ strain). ${ }^{45,46}$ Finally, histological assessment of the interface between the composite adhesive and the native nerve tissue showed penetration and entanglement of GelMA/MeTro adhesive at the interface of an anastomosed sciatic nerve
(Fig. 3D). We observe similar behavior in our previous work where MeTro was used as lung and artery adhesive. ${ }^{47}$

\section{Three dimensional encapsulation of SCs in GelMA/ MeTro composite hydrogels}

SCs play a key role in peripheral nerve regeneration, viability, proliferation, metabolic activity, and morphology in vitro. ${ }^{48-51}$ To examine the potential for GelMA/MeTro hydrogels to support peripheral glia, SCs from the sciatic nerve were used as a model cell to assess the cellular compatibility of the composite. SC viability increased from $58.85 \pm 3.79 \%$ on day 1 to $>85 \%$ by day 5 post-encapsulation (Fig. 4A, B), which is typical of SC encapsulation in collagen-based hydrogels. ${ }^{52,53} \mathrm{SCs}$ exhibited round morphologies with little spreading at day 1 postencapsulation, followed by proliferation and spreading within the matrix at day 5 (Fig. 4C). Cell proliferation was quantified by tracking the number of DAPI-stained cell nuclei over time 

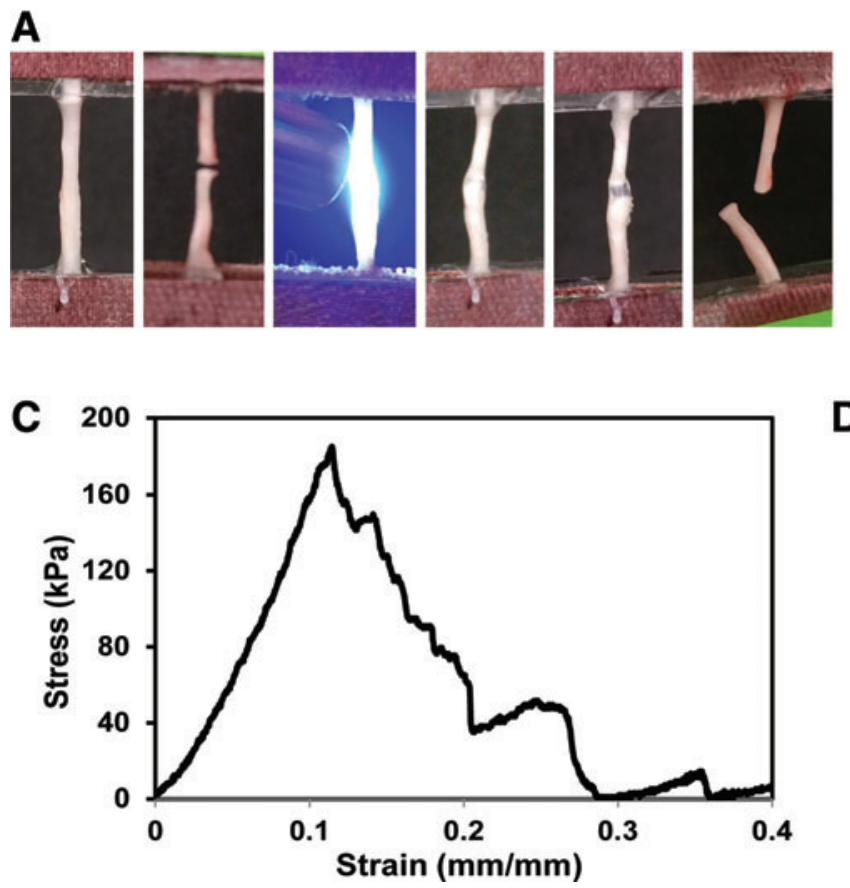

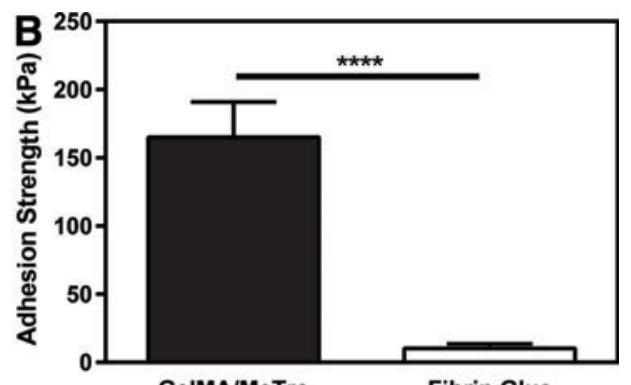

D

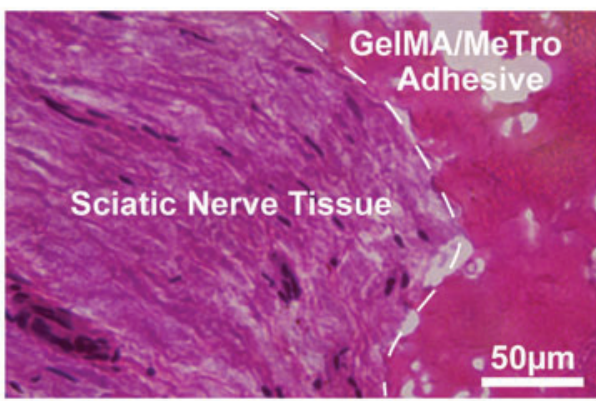

FIG. 3. Ex vivo nerve anastomosis using GelMA/MeTro hydrogel adhesives. (A) Left to right: representative photographs of clamped intact nerve with a gauge length of $12 \mathrm{~mm}$, severed nerve, photocrosslinking GelMA/MeTro composite (50/50 ratio at $10 \%$ polymer concentration) between nerve endings, anastomosed nerve, initial strain, broken nerve. (B) Adhesion strength of nerve glued by a 50/50 GelMA/MeTro composite adhesive and EVICEL ${ }^{\circledR}$ fibrin glue $(n=6)$. (C) Representative stress-strain curve of anastomosed nerves using a 50/50 GelMA/MeTro composite at $10 \%(\mathrm{w} / \mathrm{v}$ ) total polymer concentration and $180 \mathrm{~s}$ crosslinking time. (D) Representative H\&E staining from a cross-sectional area of the GelMA/MeTro (pink porous structure) composite at the interface (dashed line) of an anastomosed sciatic nerve $(* * * * p<0.0001)$. H\&E, hematoxylin and eosin. Color images available online at www.liebertpub.com/tea

(Fig. 4D). The expression of S100, a SC specific marker, also confirmed that the SC phenotype was maintained in our composites (Fig. 4E). ${ }^{54}$ A PrestoBlue assay demonstrated that the metabolic activity of the encapsulated SCs increased consistently throughout the 5 days of culture (Fig. 4F). However, both the metabolic activity and number of cells remained constant from day 1 to 3 .

\section{DRG outgrowth within GelMA/MeTro composite hydrogel}

To evaluate the ability of composite hydrogels to support neurite extension in three dimensional structures, the morphology of whole DRGs encapsulated within GelMA, MeTro, and 50/50 GelMA/MeTro (10\% [w/v] total polymer concentration) composites was characterized. DRGs were utilized to represent a heterogenous population of PNS neurons and glia (SCs). As shown in Figure 5A, robust neurite outgrowth was observed within pure GelMA hydrogels and GelMA/MeTro composites. In contrast, neurite outgrowth was hindered in pure MeTro hydrogels, likely due to the higher stiffness of MeTro. ${ }^{20}$ Despite the difference in mechanical stiffness, the quantification of neurite outgrowth $^{30}$ revealed no statistically significant differences between GelMA and 50/50 GelMA/MeTro hydrogels (Fig. 5B). Furthermore, immunofluorescent staining of outgrowth showed that the SCs endogenously found within whole DRGs were able to migrate from the encapsulated DRG within GelMA/MeTro hydrogels (Fig. 5C).

\section{Subcutaneous implantation and in vivo degradation of GelMA/MeTro hydrogel}

A murine subcutaneous implantation model was used to evaluate degradation of GelMA/MeTro in vivo. Both the weight and volume of the explanted GelMA/MeTro hydrogels decreased consistently until day 28 , followed by complete biodegradation at day 56 (Fig. 6A, B). The discrepancies between the estimations by weight and by volume are likely due to progressive tissue ingrowth within the composites (Fig. 6C, E). As expected, the in vivo degradation rate of subcutaneously implanted composites was slower than GelMA $(\sim 1 \text { week for GelMA })^{22}$ and faster than MeTro hydrogels ( $>4$ weeks for MeTro). ${ }^{34}$

As shown in Figure 6C and E, histological examination of explanted samples at days 4-28 post-implantation revealed minimal progressive tissue ingrowth. As the material degraded, a high density of cell infiltration is observed throughout the entire sample on day 28 (Fig. 6E), suggesting that autologous tissue will eventually replace the hydrogel. ${ }^{55}$ This observation of minimal immune response was further confirmed by immunofluorescent staining against surface markers representative of pro-inflammatory cell types (Fig. 6F-K). While the implanted GelMA/MeTro hydrogels are composed of both human and fish proteins, gelatin and tropoelastin are both highly conserved among different species. ${ }^{56,57}$ Furthermore, previous studies have demonstrated that decellularized matrices trigger minimal immune responses when implanted in vivo. ${ }^{58}$ Accordingly, fluorescent 

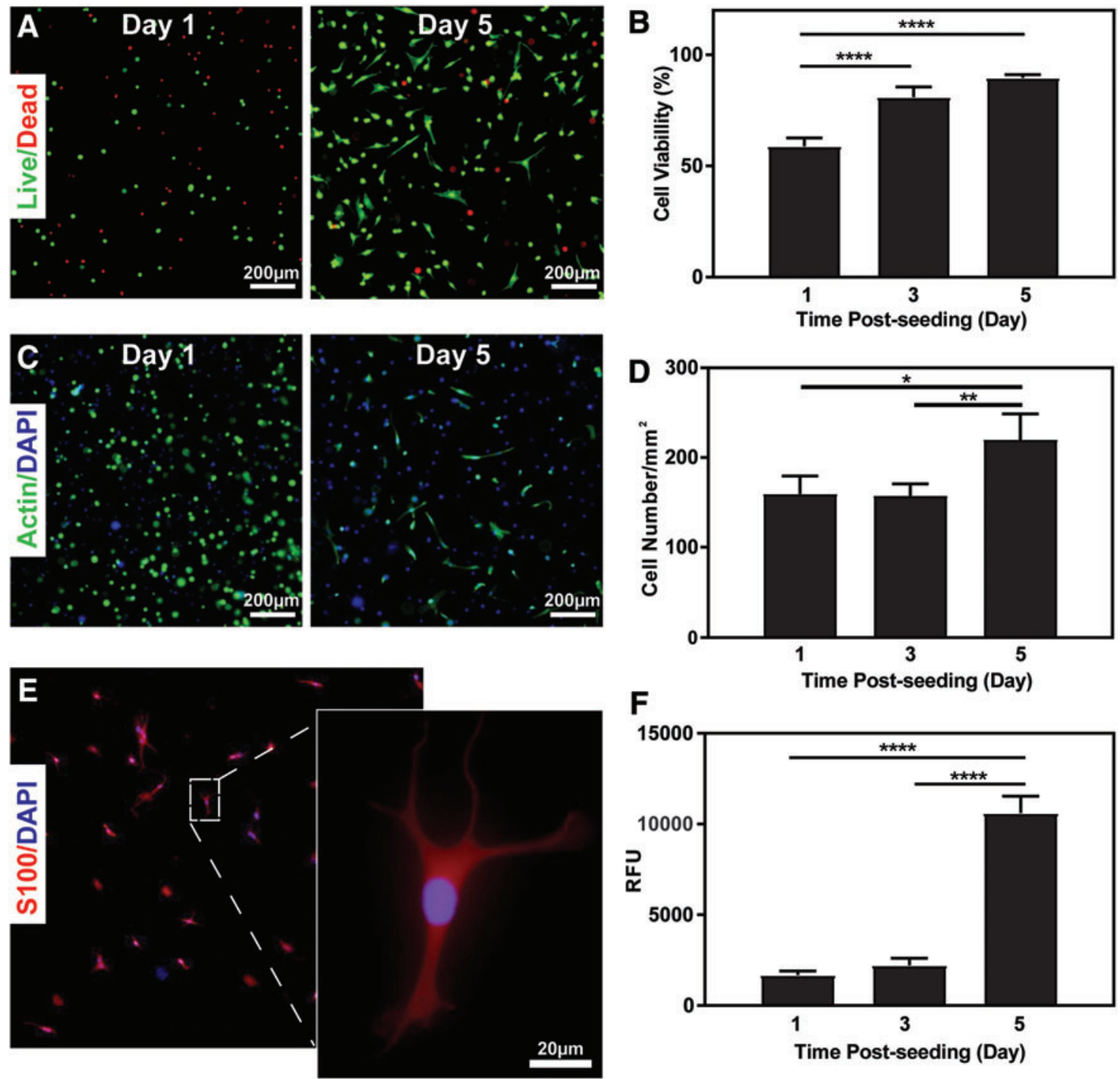

FIG. 4. In vitro SC encapsulation in a $20 / 80$ GelMA/MeTro hydrogel at $10 \%$ (w/v) total polymer concentration. (A) Representative calcein-AM (green)/ethidium homodimer (red) stained images from cell-laden hydrogels after 1 and 5 days of culture. (B) Quantification of cell viability after 1, 3, and 5 days postencapsulation. (C) Representative phalloidin (green)/DAPI (blue) stained images from cell-laden hydrogels after 1 and 5 days of encapsulation. (D) Quantification of total cell number by nuclei counting after 1,3, and 5 days of encapsulation. (E) Representative immunofluorescent image showing the expression of SC marker S100 (red)/DAPI (blue) 5 days after encapsulation. (F) Quantification of metabolic activity (RFU) by PrestoBlue 1, 3, and 5 days postencapsulation $(* p<0.05$, ** $p<0.01$, **** $p<0.0001)$. DAPI, 4',6-diamidino-2-phenylindole; RFU, relative fluorescence units. Color images available online at www.liebertpub.com/tea

images showed little T-lymphocytes (CD3) (Fig. 6F) and mild macrophage (CD68) infiltration around the implanted composite (Fig. 6I), at day 4 post-implantation. Moreover, results showed limited sustained inflammatory response, as demonstrated by the absence of CD68+ cells at day 28 postimplantation (Fig. 6H, K). These results are consistent with other recently developed elastic biomaterials, which showed complete macrophage withdrawal 28 days after implantation. ${ }^{31,32,47}$ Herein, microscopic and histological examination of subcutaneously implanted GelMA/MeTro samples revealed significant ingrowth of predominantly non-inflammatory tissue. Furthermore, these hydrogels were shown to elicit minimal immunogenicity and to be completely biodegraded at day 56 post-implantation, which is a clinically relevant time frame for PNI recovery.

\section{Discussion}

Photocrosslinkable materials may constitute a rapid strategy to reconnect nerve ends together during primary traumatic care, as opposed to secondary or revision surgical interventions. $^{44}$ The bonding strength of photocrosslinked adhesives to tissues may increase in vivo by covalent bonds generated by free radicals during the curing process. ${ }^{59}$ Furthermore, Lang et al. demonstrated that adhesive precursor materials penetrate the tissue in between the collagen fibers in the extracellular matrix (ECM) and tend to interlock following photocrosslinking. ${ }^{60}$ This in turn increases the surface area of interaction and, thus, also enhances the adhesive strength following crosslinking. Our previous results highlight the potential of our GelMA/ 

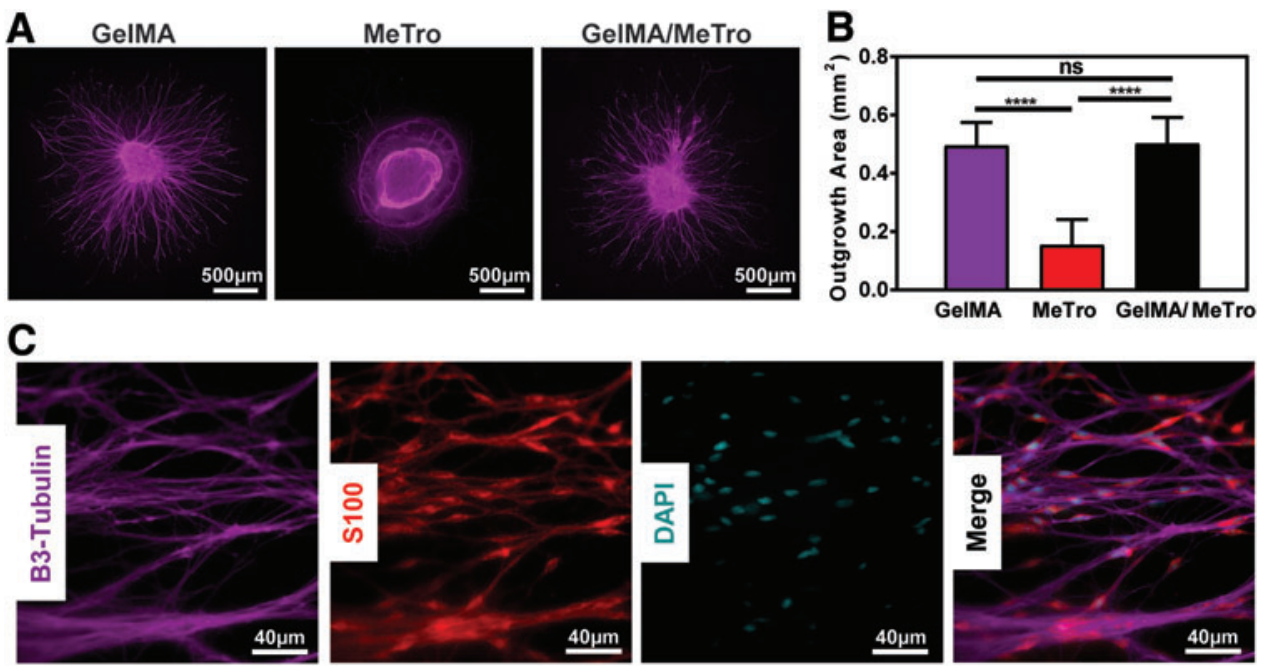

FIG. 5. In vitro dorsal root ganglia encapsulation within GelMA/MeTro hydrogels. (A) Representative $\beta$-3-tubulin ( purple) immunofluorescent images of encapsulated dorsal root ganglia in GelMA, MeTro, 50/50 GelMA/MeTro at 10\% (w/v) total polymer concentration and $30 \mathrm{~s}$ UV exposure. (B) Quantification of total outgrowth area from MATLAB image analysis $(n>4)$. (C) Representative high magnification immunofluorescent images of axonal extension in 50/50 GelMA/ MeTro at $10 \%$ (w/v) total polymer concentration, $\beta$-3-tubulin, and SC morphology, S100 (red), and cell nuclei (cyan). (ns: $p>0.05, * * * * p<0.0001)$. Color images available online at www.liebertpub.com/tea

MeTro composite as a bioadhesive. ${ }^{32}$ In particular, GelMA/ MeTro composites exhibited adhesive strength significantly greater than other commercially available fibrin-based adhesives. ${ }^{32}$ In this study, we aimed to further improve the mechanical and adhesive properties of our composite hydrogel adhesive by switching to a UV photocrosslinking system and a type I photoinitiator, which aims to reduce the use of sutures by providing high adhesion to neural tissue. It has been demonstrated that an optimized duration and intensity of UV light has no harmful effects for the encapsulated cells. ${ }^{61,62}$ In this study, SCs encapsulated within UV crosslinked GelMA/MeTro composite proliferated and recovered, which indicates the potential of the hydrogel to serve as a biomaterial-based vehicle for SC delivery.

As an initial proof-of-principle test, an ex vivo evaluation of nerve anastomosis of murine sciatic nerves highlighted the potential of this material for sutureless repair (Fig. 3). In this study, the composite precursor is likely to form interactions with the individual fascicles within the nerve, increasing the total surface area in contact with the tissue after crosslinking and, thus, the overall shear forces during extension. ${ }^{63}$ This hypothesis was supported by the gradual failure of the adhesive in the stress-strain curves (Fig. 3C), similar to that observed for intact nerves. ${ }^{64}$ In addition, GelMA/MeTro adhesive may be capable of withstanding the physiological levels of strain $(\sim 10 \%)$ for immobilized extremities following PNI, ${ }^{45,46}$ which is common for the treatment of musculoskeletal injuries. ${ }^{6,66}$ The rapid solidification of our material may enable nerve repair to be carried out at the initial point of care, reducing the number of surgical revisions. To further improve the mechanical stability of GelMA/MeTro as a neural adhesive, as well as reducing the risk of neuroma formation, our material may be used in combination with neural wraps and conduits to provide structural cues for axonal regeneration. ${ }^{67}$ In this case, a combinatorial design using a pure MeTro hydrogel as a neural wrap may reduce the risk of neuromas by preventing neurite extension and isolating the injury site from surrounding tissue.

In addition, our GelMA/MeTro composite system may be well suited for glial support cell delivery as the cell-laden hydrogel precursor solutions possess a low viscosity, ${ }^{68}$ ensuring low shear stress during injection, and homogenous distribution of SC payload. $^{69}$ The PNS has a remarkable regenerative potential compared to the central nervous system. ${ }^{2}$ However, this regenerative ability is insufficient for large gap injuries $(>4 \mathrm{~cm})$, since the axonal growth rate has been shown to only be $\sim 1 \mathrm{~mm} /$ day. ${ }^{70}$ A key rate limiting component of PNS regeneration of large-gap injuries is the participation of supportive glia, SCs. SCs express neural adhesion membrane proteins (NCAM, L1CAM, and N-cadherin), ${ }^{71}$ secrete neurotropic factors, ${ }^{72}$ and generate ECM proteins, which promote neurite extension. ${ }^{73}$

Autografts remain the preferred treatment as they provide not only a structured conduit for axonal guidance but also possess a homogenous distribution of autologous SCs. Thus, $\mathrm{SC}$ inclusion must be taken into consideration for the design of synthetic alternatives as the inclusion of exogenous SCs within these conduits was shown to be a promising strategy to increase the rate of nerve regeneration. ${ }^{52,67,74}$ Although the tensile moduli of the engineered composites herein were below those of native neural tissues $(\sim 22.7 \mathrm{MPa}),{ }^{44} \mathrm{GelMA} /$ MeTro hydrogels provided a neuro-supportive environment, which may enhance regeneration following an injury. In this study, encapsulated SCs in GelMA/MeTro hydrogels displayed a characteristic elongated morphology (Fig. 4) after an initial quiescent period following encapsulation, an improvement over alginate-based hydrogel, which was previously used for encapsulation and delivery of SCs. ${ }^{75,76}$ Encapsulated $\mathrm{SCs}$ exhibited round morphologies in alginate due to an absence of cell adhesion domains, ultimately leading to atypical behavior. Both gelatin and tropoelastin are likely to promote SC adhesion because of cell-binding domains within their structure. ${ }^{77}$ GelMA hydrogels have been shown to support the growth and proliferation of encapsulated cells, due to the 

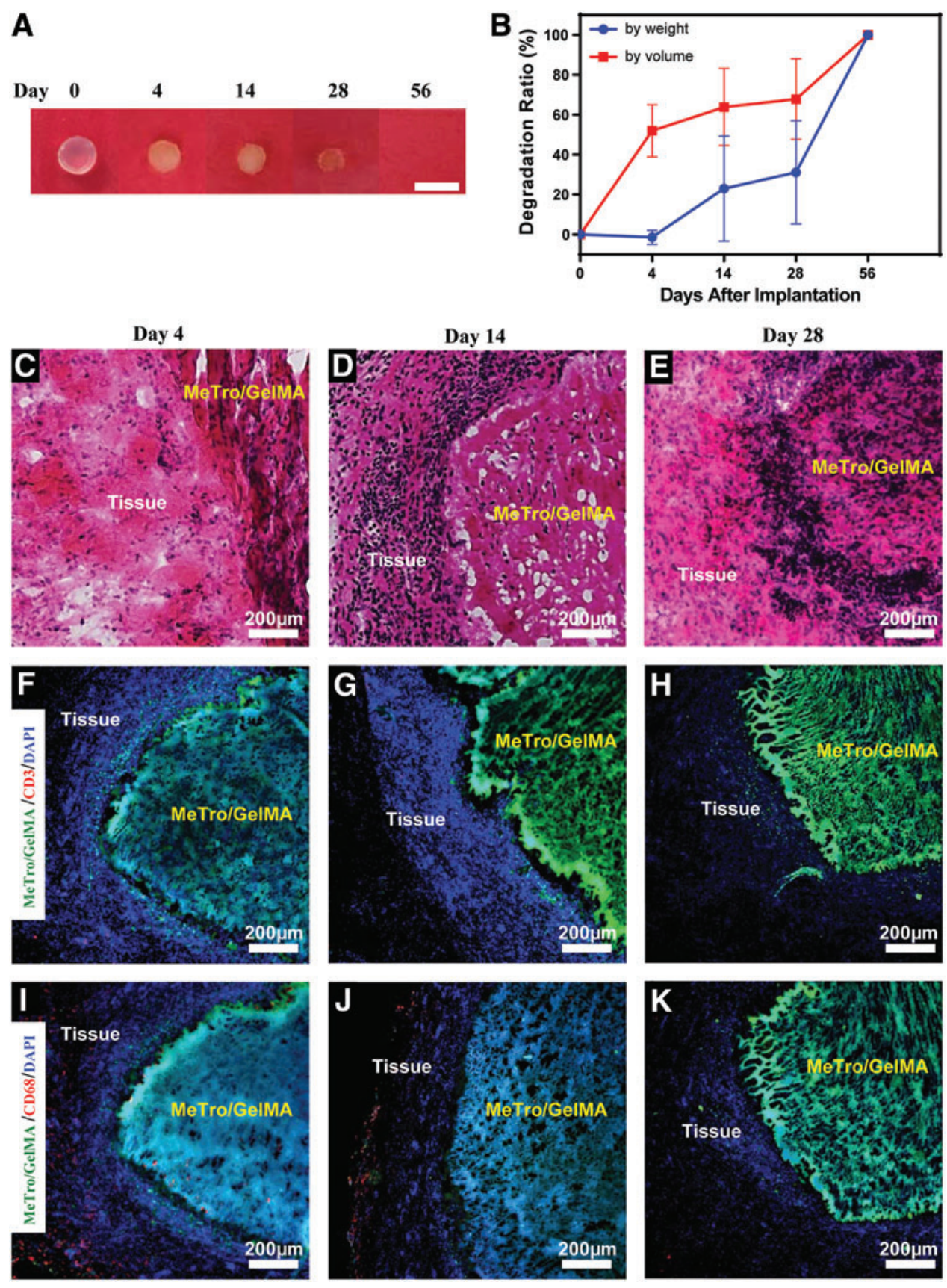

FIG. 6. In vivo biocompatibility and biodegradation of GelMA/MeTro composite hydrogel using a rat subcutaneous model. (A) Representative images of GelMA/MeTro hydrogels on days 0, 4, 14, 28, and 56 post-implantation. (B) In vivo degradation of GelMA/MeTro hydrogels based on weight and volume loss of the implants $(n=3)$. H\&E staining of GelMA/ MeTro hydrogels with the surrounding tissue (C) 4, (D) 14, and (E) 28 days post-implantation, showing tissue integration. Immunofluorescent staining of subcutaneously implanted GelMA/MeTro hydrogels demonstrating no significant local lymphocyte infiltration (CD3, red) at days (F) 4, (G) 14, and (H) 28. Immunofluorescent staining against macrophages $($ CD68, red) at day (I) 4 shows a significant reduction after day $(\mathbf{J}) 14$ and $(\mathbf{K})$ 28. GelMA/MeTro composites are green (auto fluorescent), and cell nuclei (DAPI) are in blue. Color images available online at www.liebertpub.com/tea

presence of Arg-Gly-Asp (RGD) domains. ${ }^{19}$ In addition, SCs have also been shown to express $\alpha v \beta 3$ integrins, which function as cell-binding cites within tropoelastin. ${ }^{78,79}$

While GelMA was less stiff than MeTro, pure GelMA hydrogels contain several matrix metalloproteinase (MMP)sensitive degradation sequences, ${ }^{80}$ exposing them to more rapid degradation, reduced tissue integration, and change in bulk mechanical properties in vivo. ${ }^{22}$ These dynamic properties following implantation are bolstered by SC over-expression of MMPs to remove cellular debris following PNI, ${ }^{81,82}$ which may further expedite degradation. A slower degradation rate achieved with higher loading fractions of MeTro may provide a suitable isolated microenvironment to protect the injury site from fibrosis and inflammation during regeneration. ${ }^{13,83}$ Furthermore, in our recent work, we have shown that MeTro hydrogels can adhere to the tissue due to physical interaction 
and interlocking, as well as charge interactions (positively charged tropoelastin and negatively charged glycosaminoglycans on the ECM) ${ }^{47}$ In addition, MeTro coacervates at body temperature, ${ }^{20}$ making the precursor solution hydrophobic following injection; therefore, the material will stay confined to the site of application. ${ }^{47}$ All these unique properties of MeTro, in combination with the high biocompatibility of GelMA, could support neurite extension from encapsulated DRGs in vitro (Fig. 5) and may ultimately enhance the rate of peripheral nerve regeneration in vivo.

Successful repair in large-gap injuries with off-the-shelf solutions requires both guidance cues and SC participation to support outgrowing axons traversing the coaptation site. ${ }^{84}$ Previously, hydrogels reported to promote neurite outgrowth of DRGs have shown limited SC migration within scaffolds. ${ }^{85}$ However, Gonzalez-Perez et al. demonstrated that collagen-based hydrogels used as fillers for neural conduits led to increased muscle reinnervation and myelination. ${ }^{86}$ Therefore, optimized material formulations of GelMA/MeTro hydrogels may be used in conjunction with delivery of exogenous SCs to increase the efficacy of treating large gap injuries with allografts. As a material capable of delivering SCs, supporting and preventing neurite extension, and providing mechanical stability, GelMA/MeTro composites may be used to overcome the limitations of currently available materials and therapies used for the clinical management of PNI and necessitate further investigation on function recovery in vivo.

\section{Conclusion}

In this study, we engineered a highly biocompatible GelMA/MeTro hydrogel adhesive with tunable physical properties for sutureless nerve repair and/or delivery SCs toward the clinical management of PNI. The mechanical properties, swelling ratios, and degradation rates of the engineered composites were modulated by varying the polymer ratios, total polymer concentrations, and crosslinking time. GelMA/ MeTro composites exhibited higher failure strength for bonded sciatic nerves compared to clinically used fibrin-based adhesives. Furthermore, GelMA/MeTro hydrogels supported the proliferation and spreading of encapsulated glia in vitro. The composites provided a supportive microenvironment for neurite extension, as demonstrated by the robust neurite outgrowth of encapsulated DRGs. Finally, the composites were shown to be biocompatible and biodegradable in vivo, as confirmed by subcutaneous implantation in a murine animal model. Taken together, these results show that GelMA/ MeTro hydrogels hold remarkable potential for regenerative nerve tissue engineering applications for the treatment of PNI.

\section{Acknowledgments}

N.A. acknowledges the support from the American Heart Association (AHA, 16SDG31280010), the National Institutes of Health (NIH, R01-EB023052; and NIH, R01HL140618), and FY17 TIER 1 Interdisciplinary Research Seed Grants. R.A.K. and N.A. acknowledge Northeastern University and the startup fund provided by the Department of Chemical Engineering, College of Engineering at Northeastern University.

\section{Author Contributions}

J.R.S., R.A.K., and N.A. conceived the project. J.R.S. and R.A.K. isolated neonatal rat DRGs. J.R.S. and A.N.K. isolated adult rat sciatic nerves. J.R.S. completed the nerve anastomosis experiments and the in vitro cell studies with SCs and DRGs. J.R.S. and E.S.S. performed in vivo animal surgeries. E.S.S. completed the SEM and NMR work. D.D. isolated and purified SCs from neonatal rats under the advisement of A.N.K. R.P.L. and E.S.S. performed the H\&E staining. F.D. measured the physical properties of the materials under the supervision of N.A. A.S.W. and A.N.K. provided intellectual input and advice. J.R.S., R.A.K., and N.A. analyzed the results, prepared the figures, and wrote the article. J.R.S., E.S.S., D.D., R.P.L., A.S.W., A.N.K., R.A.K., and N.A. edited the article. R.A.K. and N.A. supervised the work.

\section{Disclosure Statement}

A.S.W. is the Scientific Founder of Elastagen Pty Ltd.

\section{References}

1. Bhangra, K.S., Busuttil, F., Phillips, J.B., and Rahim, A.A. Using stem cells to grow artificial tissue for peripheral nerve repair. Stem Cells Int 2016, 7502178, 2016.

2. Zhou, Y., and Notterpek, L. Promoting peripheral myelin repair. Exp Neurol 283, 573, 2016.

3. Wolford, L.M., and Stevao, E.L. Considerations in nerve repair. Proc (Bayl Univ Med Cent) 16, 152, 2003.

4. Kehoe, S., Zhang, X.F., and Boyd, D. FDA approved guidance conduits and wraps for peripheral nerve injury: a review of materials and efficacy. Injury 43, 553, 2012.

5. Chuang, T.H., Wilson, R.E., Love, J.M., Fisher, J.P., and Shah, S.B. A novel internal fixator device for peripheral nerve regeneration. Tissue Eng Part C Methods 19, 427, 2013.

6. Schmidhammer, R., Zandieh, S., Hopf, R., et al. Alleviated tension at the repair site enhances functional regeneration: the effect of full range of motion mobilization on the regeneration of peripheral nerves - histologic, electrophysiologic, and functional results in a rat model. J Trauma 56, 571, 2004.

7. Menovsky, T., and Beek, J.F. Laser, fibrin glue, or suture repair of peripheral nerves: a comparative functional, histological, and morphometric study in the rat sciatic nerve. $\mathrm{J}$ Neurosurg 95, 694, 2001.

8. Menovsky, T., Beek, J.F., and Thomsen, S.L. Laser(-assisted) nerve repair. A review. Neurosurg Rev 18, 225, 1995.

9. Johnson, T.S., O'Neill, A.C., Motarjem, P.M., et al. Photochemical tissue bonding: a promising technique for peripheral nerve repair. J Surg Res 143, 224, 2007.

10. O'Neill, A.C., Randolph, M.A., Bujold, K.E., Kochevar, I.E., Redmond, R.W., and Winograd, J.M. Photochemical sealing improves outcome following peripheral neurorrhaphy. J Surg Res 151, 33, 2009.

11. Landegren, T., Risling, M., Brage, A., and Persson, J.K. Long-term results of peripheral nerve repair: a comparison of nerve anastomosis with ethyl-cyanoacrylate and epineural sutures. Scand J Plast Reconstr Surg Hand Surg 40, 65, 2006.

12. Barton, M.J., Morley, J.W., Stoodley, M.A., Lauto, A., and Mahns, D.A. Nerve repair: toward a sutureless approach. Neurosurg Rev 37, 585, 2014.

13. Lauto, A., Mawad, D., and Foster, L.J.R. Adhesive biomaterials for tissue reconstruction. J Chem Technol Biotechnol 83, 464, 2008.

14. Ornelas, L., Padilla, L., Di Silvio, M., et al. Fibrin glue: an alternative technique for nerve coaptation-part I. Wave amplitude, conduction velocity, and plantar-length factors. J Reconstr Microsurg 22, 119, 2006. 
15. Ornelas, L., Padilla, L., Di Silvio, M., et al. Fibrin glue: an alternative technique for nerve coaptation-part II. Nerve regeneration and histomorphometric assessment. J Reconstr Microsurg 22, 123, 2006.

16. Temple, C.L., Ross, D.C., Dunning, C.E., and Johnson, J.A. Resistance to disruption and gapping of peripheral nerve repairs: an in vitro biomechanical assessment of techniques. J Reconstr Microsurg 20, 645, 2004.

17. Wieken, K., Angioi-Duprez, K., Lim, A., Marchal, L., and Merle, M. Nerve anastomosis with glue: comparative histologic study of fibrin and cyanoacrylate glue. J Reconstr Microsurg 19, 17, 2003.

18. Kline, D.G., and Hayes, G.J. An experimental evaluation of the effect of a plastic adhesive, methyl 2-cyanoacrylate, on neural tissue. J Neurosurg 20, 647, 1963.

19. Nichol, J.W., Koshy, S.T., Bae, H., Hwang, C.M., Yamanlar, S., and Khademhosseini, A. Cell-laden microengineered gelatin methacrylate hydrogels. Biomaterials 31, 5536, 2010.

20. Annabi, N., Mithieux, S.M., Zorlutuna, P., Camci-Unal, G., Weiss, A.S., and Khademhosseini, A. Engineered cell-laden human protein-based elastomer. Biomaterials 34, 5496, 2013.

21. Wende, F.J., Gohil, S., Nord, L.I., Helander Kenne, A., and Sandstrom, C. 1D NMR methods for determination of degree of cross-linking and BDDE substitution positions in HA hydrogels. Carbohydr Polym 157, 1525, 2017.

22. Chen, Y.C., Lin, R.Z., Qi, H., et al. Functional human vascular network generated in photocrosslinkable gelatin methacrylate hydrogels. Adv Funct Mater 22, 2027, 2012.

23. Koppes, A.N., Keating, K.W., McGregor, A.L., et al. Robust neurite extension following exogenous electrical stimulation within single walled carbon nanotube-composite hydrogels. Acta Biomater 39, 34, 2016.

24. Brewer, G.J., and Price, P.J. Viable cultured neurons in ambient carbon dioxide and hibernation storage for a month. Neuroreport 7, 1509, 1996.

25. Koppes, A.N., Nordberg, A.L., Paolillo, G.M., et al. Electrical stimulation of Schwann cells promotes sustained increases in neurite outgrowth. Tissue Eng Part A 20, 494, 2014.

26. Behan, B.L., DeWitt, D.G., Bogdanowicz, D.R., Koppes, A.N., Bale, S.S., and Thompson, D.M. Single-walled carbon nanotubes alter Schwann cell behavior differentially within 2D and 3D environments. J Biomed Mater Res A 96, 46, 2011.

27. Koppes, A.N., Seggio, A.M., and Thompson, D.M. Neurite outgrowth is significantly increased by the simultaneous presentation of Schwann cells and moderate exogenous electric fields. J Neural Eng 8, 046023, 2011.

28. Koppes, A.N., Zaccor, N.W., Rivet, C.J., et al. Neurite outgrowth on electrospun PLLA fibers is enhanced by exogenous electrical stimulation. J Neural Eng 11, 046002, 2014.

29. Koppes, R.A., Park, S., Hood, T., et al. Thermally drawn fibers as nerve guidance scaffolds. Biomaterials 81, 27, 2016.

30. Park, S., Koppes, R.A., Froriep, U.P., et al. Optogenetic control of nerve growth. Sci Rep 5, 9669, 2015.

31. Zhang, Y.N., Avery, R.K., Vallmajo-Martin, Q., et al. A highly elastic and rapidly crosslinkable elastin-like polypeptide-based hydrogel for biomedical applications. Adv Funct Mater 25, 4814, 2015.

32. Annabi, N., Rana, D., Shirzaei Sani, E., et al. Engineering a sprayable and elastic hydrogel adhesive with antimicrobial properties for wound healing. Biomaterials 139, 229, 2017.

33. Noshadi, I., Walker, B.W., Portillo-Lara, R., et al. Engineering biodegradable and biocompatible bio-ionic liquid conjugated hydrogels with tunable conductivity and mechanical properties. Sci Rep 7, 4345, 2017.
34. Annabi, N., Shin, S.R., Tamayol, A., et al. Highly elastic and conductive human-based protein hybrid hydrogels. Adv Mater 28, 40, 2016.

35. Lai, T.C., Yu, J., and Tsai, W.B. Gelatin methacrylate/carboxybetaine methacrylate hydrogels with tunable crosslinking for controlled drug release. J Mater Chem B 4, 2304, 2016.

36. Annabi, N., Nichol, J.W., Zhong, X., et al. Controlling the porosity and microarchitecture of hydrogels for tissue engineering. Tissue Eng Part B Rev 16, 371, 2010.

37. Zhu, J., and Marchant, R.E. Design properties of hydrogel tissueengineering scaffolds. Expert Rev Med Devices 8, 607, 2011.

38. Khaing, Z.Z., Ehsanipour, A., Hofstetter, C.P., and Seidlits, S.K. Injectable hydrogels for spinal cord repair: a focus on swelling and intraspinal pressure. Cells Tissues Organs 202, 67, 2016.

39. Nectow, A.R., Marra, K.G., and Kaplan, D.L. Biomaterials for the development of peripheral nerve guidance conduits. Tissue Eng Part B Rev 18, 40, 2012.

40. Schuurman, W., Levett, P.A., Pot, M.W., et al. Gelatinmethacrylamide hydrogels as potential biomaterials for fabrication of tissue-engineered cartilage constructs. Macromol Biosci 13, 551, 2013.

41. Van Den Bulcke, A.I., Bogdanov, B., De Rooze, N., Schacht, E.H., Cornelissen, M., and Berghmans, H. Structural and rheological properties of methacrylamide modified gelatin hydrogels. Biomacromolecules 1, 31, 2000.

42. Lam, J., Kim, K., Lu, S., et al. A factorial analysis of the combined effects of hydrogel fabrication parameters on the in vitro swelling and degradation of oligo(poly(ethylene glycol) fumarate) hydrogels. J Biomed Mater Res A 102, 3477, 2014.

43. Grinsell, D., and Keating, C.P. Peripheral nerve reconstruction after injury: a review of clinical and experimental therapies. Biomed Res Int 2014, 698256, 2014.

44. Rickett, T., Amoozgar, Z., Sun, W.J., Yeo, Y., and Shi, R.Y. A photo-crosslinkable chitosan hydrogel for peripheral nerve anastomosis. Int Conf Biomed 1078, 2009.

45. Li, J., and Shi, R. Stretch-induced nerve conduction deficits in guinea pig ex vivo nerve. J Biomech 40, 569, 2007.

46. Jou, I.M., Lai, K.A., Shen, C.L., and Yamano, Y. Changes in conduction, blood flow, histology, and neurological status following acute nerve-stretch injury induced by femoral lengthening. J Orthop Res 18, 149, 2000.

47. Annabi, N., Zhang, Y.N., Assmann, A., et al. Engineering a highly elastic human protein-based sealant for surgical applications. Sci Transl Med 9, pii: eaai7466, 2017.

48. Salzer, J.L. Schwann cell myelination. Cold Spring Harb Perspect Biol 7, a020529, 2015.

49. Bozkurt, A., Deumens, R., Beckmann, C., et al. In vitro cell alignment obtained with a Schwann cell enriched microstructured nerve guide with longitudinal guidance channels. Biomaterials 30, 169, 2009.

50. Madduri, S., and Gander, B. Schwann cell delivery of neurotrophic factors for peripheral nerve regeneration. $\mathrm{J}$ Peripher Nerv Syst 15, 93, 2010.

51. Schmidt, C.E., and Leach, J.B. Neural tissue engineering: strategies for repair and regeneration. Annu Rev Biomed Eng 5, 293, 2003.

52. Suri, S., and Schmidt, C.E. Cell-laden hydrogel constructs of hyaluronic acid, collagen, and laminin for neural tissue engineering. Tissue Eng Part A 16, 1703, 2010.

53. Dewitt, D.D., Kaszuba, S.N., Thompson, D.M., and Stegemann, J.P. Collagen I-matrigel scaffolds for enhanced Schwann cell survival and control of three-dimensional cell morphology. Tissue Eng Part A 15, 2785, 2009. 
54. Liu, Z., Jin, Y.Q., Chen, L., et al. Specific marker expression and cell state of Schwann cells during culture in vitro. PLoS One 10, e0123278, 2015.

55. Nguyen, K.T., and West, J.L. Photopolymerizable hydrogels for tissue engineering applications. Biomaterials 23, 4307, 2002.

56. Stover, D.A., and Verrelli, B.C. Comparative vertebrate evolutionary analyses of type I collagen: potential of COL1a1 gene structure and intron variation for common bonerelated diseases. Mol Biol Evol 28, 533, 2011.

57. Boyd, C.D., Christiano, A.M., Pierce, R.A., Stolle, C.A., and Deak, S.B. Mammalian tropoelastin: multiple domains of the protein define an evolutionarily divergent amino acid sequence. Matrix 11, 235, 1991.

58. Cheng, C.W., Solorio, L.D., and Alsberg, E. Decellularized tissue and cell-derived extracellular matrices as scaffolds for orthopaedic tissue engineering. Biotechnol Adv 32, 462, 2014.

59. Yao, M., Yaroslavsky, A., Henry, F.P., Redmond, R.W., and Kochevar, I.E. Phototoxicity is not associated with photochemical tissue bonding of skin. Lasers Surg Med 42, 123, 2010.

60. Lang, N., Pereira, M.J., Lee, Y., et al. A blood-resistant surgical glue for minimally invasive repair of vessels and heart defects. Sci Transl Med 6, 218ra6, 2014.

61. Annabi, N., Tamayol, A., Uquillas, J.A., et al. 25th Anniversary article: rational design and applications of hydrogels in regenerative medicine. Adv Mater 26, 85, 2014.

62. Loessner, D., Meinert, C., Kaemmerer, E., et al. Functionalization, preparation and use of cell-laden gelatin methacryloyl-based hydrogels as modular tissue culture platforms. Nat Protoc 11, 727, 2016.

63. Flores, A.J., Lavernia, C.J., and Owens, P.W. Anatomy and physiology of peripheral nerve injury and repair. Am J Orthop (Belle Mead NJ) 29, 167, 2000.

64. Borschel, G.H., Kia, K.F., Kuzon, W.M., Jr., and Dennis, R.G. Mechanical properties of acellular peripheral nerve. J Surg Res 114, 133, 2003.

65. Vipond, N., Taylor, W., and Rider, M. Postoperative splinting for isolated digital nerve injuries in the hand. $\mathbf{J}$ Hand Ther 20, 222, 2007.

66. Rempel, D., Dahlin, L., and Lundborg, G. Pathophysiology of nerve compression syndromes: response of peripheral nerves to loading. J Bone Joint Surg Am 81, 1600, 1999.

67. Pabari, A., Yang, S.Y., Mosahebi, A., and Seifalian, A.M. Recent advances in artificial nerve conduit design: strategies for the delivery of luminal fillers. J Control Release 156, 2, 2011.

68. Paul, A., Hasan, A., Kindi, H.A., et al. Injectable graphene oxide/hydrogel-based angiogenic gene delivery system for vasculogenesis and cardiac repair. ACS Nano 8, 8050, 2014.

69. Patel, V., Joseph, G., Patel, A., et al. Suspension matrices for improved Schwann-cell survival after implantation into the injured rat spinal cord. J Neurotrauma 27, 789, 2010.

70. Jonsson, S., Wiberg, R., McGrath, A.M., et al. Effect of delayed peripheral nerve repair on nerve regeneration, Schwann cell function and target muscle recovery. PLoS One 8, e56484, 2013.

71. Bixby, J.L., Lilien, J., and Reichardt, L.F. Identification of the major proteins that promote neuronal process outgrowth on Schwann cells in vitro. J Cell Biol 107, 353, 1988.

72. Terenghi, G. Peripheral nerve regeneration and neurotrophic factors. J Anat 194 (Pt 1), 1, 1999.

73. Romano, N.H., Madl, C.M., and Heilshorn, S.C. Matrix RGD ligand density and L1CAM-mediated Schwann cell interactions synergistically enhance neurite outgrowth. Acta Biomater 11, 48, 2015.
74. Mosahebi, A., Simon, M., Wiberg, M., and Terenghi, G. A novel use of alginate hydrogel as Schwann cell matrix. Tissue Eng 7, 525, 2001.

75. Novikova, L.N., Mosahebi, A., Wiberg, M., Terenghi, G., Kellerth, J.O., and Novikov, L.N. Alginate hydrogel and matrigel as potential cell carriers for neurotransplantation. J Biomed Mater Res A 77, 242, 2006.

76. Wang, Y., Zhang, G., Hou, Y., et al. Transplantation of microencapsulated Schwann cells and mesenchymal stem cells augment angiogenesis and improve heart function. Mol Cell Biochem 366, 139, 2012.

77. White, J.D., Wang, S., Weiss, A.S., and Kaplan, D.L. Silktropoelastin protein films for nerve guidance. Acta Biomater 14, 1, 2015.

78. Milner, R., Wilby, M., Nishimura, S., et al. Division of labor of Schwann cell integrins during migration on peripheral nerve extracellular matrix ligands. Dev Biol 185, 215, 1997.

79. Bax, D.V., Rodgers, U.R., Bilek, M.M., and Weiss, A.S. Cell adhesion to tropoelastin is mediated via the C-terminal GRKRK motif and integrin alphaVbeta3. J Biol Chem 284, 28616, 2009.

80. Vandooren, J., Van den Steen, P.E., and Opdenakker, G. Biochemistry and molecular biology of gelatinase B or matrix metalloproteinase-9 (MMP-9): the next decade. Crit Rev Biochem Mol Biol 48, 222, 2013.

81. Liu, H., Kim, Y., Chattopadhyay, S., Shubayev, I., Dolkas, J., and Shubayev, V.I. Matrix metalloproteinase inhibition enhances the rate of nerve regeneration in vivo by promoting dedifferentiation and mitosis of supporting schwann cells. J Neuropathol Exp Neurol 69, 386, 2010.

82. Parrinello, S., Napoli, I., Ribeiro, S., et al. EphB signaling directs peripheral nerve regeneration through Sox2dependent Schwann cell sorting. Cell 143, 145, 2010.

83. Sameem, M., Wood, T.J., and Bain, J.R. A systematic review on the use of fibrin glue for peripheral nerve repair. Plast Reconstr Surg 127, 2381, 2011.

84. Son, Y.J., and Thompson, W.J. Schwann cell processes guide regeneration of peripheral axons. Neuron 14, 125, 1995.

85. Lampe, K.J., Antaris, A.L., and Heilshorn, S.C. Design of three-dimensional engineered protein hydrogels for tailored control of neurite growth. Acta Biomater 9, 5590, 2013.

86. Gonzalez-Perez, F., Cobianchi, S., Heimann, C., Phillips, J.B., Udina, E., and Navarro, X. Stabilization, rolling, and addition of other extracellular matrix proteins to collagen hydrogels improve regeneration in chitosan guides for long peripheral nerve gaps in rats. Neurosurgery 80, 465, 2017.

Address correspondence to: Ryan A. Koppes

Department of Chemical Engineering Northeastern University Boston, MA 02115

E-mail: r.koppes@northeastern.edu

Nasim Annabi

Department of Chemical Engineering

Northeastern University

Boston, MA 02115

E-mail: n.annabi@northeastern.edu

Received: December 4, 2017

Accepted: March 21, 2018

Online Publication Date: May 8, 2018 